\title{
Modeling Light-Extraction Characteristics of Packaged Light-Emitting Diodes
}

\author{
D. Z.-Y. TING ${ }^{\mathrm{a}, \mathrm{b}, *}$ and T. C. MCGILL ${ }^{\mathrm{b}, \boldsymbol{\dagger}}$ \\ ${ }^{a}$ Thomas J. Watson, Sr., Laboratory of Applied Physics, California Institute of Technology Pasadena, California 91125, USA; \\ ${ }^{b}$ Department of Physics, National Tsing Hua University, Hsinchu, Taiwan 300, ROC
}

\begin{abstract}
We employ a Monte Carlo ray-tracing technique to model light-extraction characteristics of light-emitting diodes. By relaxing restrictive assumptions on photon traversal history, our method improves upon available analytical models for estimating light-extraction efficiencies from bare LED chips, and enhances modeling capabilities by realistically treating the various processes which photons can encounter in a packaged LED. Our method is not only capable of calculating extraction efficiencies, but can also provide extensive statistical information on photon extraction processes, and predict LED spatial emission characteristics.
\end{abstract}

Keywords: LED photon extraction, LED efficiency

\section{INTRODUCTION}

Analytical models[1,2] developed in the past have been very useful for considering photon extraction from semiconductor LED chips in limiting cases. Taking advantage of high-speed computation platforms available today, we approach the problem with a numerical Monte Carlo ray-tracing technique which promises greater range of validity. Photon extraction from LED chips is difficult for several reasons. Absorption by the medium and contacts prevent many of the internally generated photons from ever reaching exposed chip surfaces in the first place. A substantial fraction of the photons that do reach the surface end up being internally reflected because critical angles at chip surfaces are typically small due to the large index of refraction mismatch between the semiconductor LED chip and its surrounding medium. Although each photon may encounter a number of these processes, it is straight-forward to keep track of photon traversal history numerically. Therefore Monte Carlo techniques well-suited for simulating photon extraction.

\section{MODEL}

Figure 1 illustrates the T-1 3/4 LED lamp, which is used as the prototype structure in our Monte Carlo particle simulation. The particles used are classical photons (corpuscles) which obey the rules of geometrical optics. A simulation is performed with an ensemble of randomly generated photons, and the processes which each photon encounters (e.g., medium absorption, transmission/reflection at interfaces) are governed by probabilistic models. We

\footnotetext{
* Tel: 886.35.715131x3246. Fax: 886.35.723052. Email: dzt@phys.nthu.tw

$†$ Corresponding author. Tel: (818) 395-4849. Fax: (818) 568-8972. Email: tcm@ssdp.caltech.edu
} 


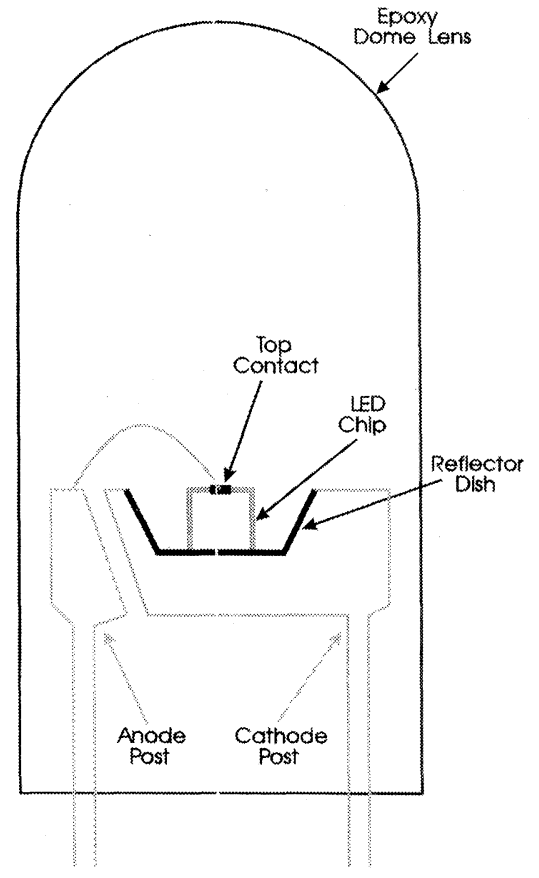

FIGURE 1 Illustration of the T-1 3/4 LED lamp (not to scale) used in our simulations. Note that the anode post, cathode post, and the bonding wire are ignored in our simulations

assume that in the LED chip photons are generated uniformly within a thin planar region at a height of $z=h_{\text {junct }}$ from the base of the chip, and are emitted in an isotropic spatial pattern.[1] We also assume monochromatic photon emission for simplicity. A trivial extension to our model would allow for the treatment of more general emission spectra. For each photon, a position vector on the emission plane and a direction vector are randomly generated. A ray-polygon intersection algorithm[3] is used to compute the intersection of photon trajectory with one of the six chip surfaces. Since a photon might be absorbed inside the chip before reaching the destination surface, we use a Monte Carlo routine to determine whether absorption takes place en route. Let the path length between the initial and final positions be $\Delta$. The probability of a photon being absorbed in a distance $\Delta$ is given by

$$
P(\Delta)=1-e^{-\alpha \Delta},
$$

where $\alpha$ is the medium absorption coefficient. We generate a random number $R$ between 0 and 1 ; absorption takes place if $R \leq P(\Delta)$. If a photon reaches a destination chip surface without being absorbed by the semiconductor, we check to see if it strikes either the top or the bottom contact, where contact absorption processes can occur. For simplicity, we assume that the contacts are totally absorbing, although partially absorbing contacts can be modeled quite easily. If the photon is not absorbed, we determine whether it is transmitted or reflected by generating a random number between 0 and 1 , and comparing it to the transmission probability given by the Fresnel equations.[4] An internally reflected photon continues on its path within the LED chip until it is eventually absorbed or transmitted. A photon emitted by the LED chip is then ray-traced to the reflector dish, and then to the epoxy lens. For speed and simplicity, we assume that the three stages of the simulation occur sequentially (chip, then reflector, then lens). Therefore photon re-entrance into the LED chip is not considered, nor the possibility of internally reflected photons in the lens re-striking the reflector dish. The error associated with the sequential approximations has been estimated to be a few percent. Throughout the different stages of the simulation, extensive statistics are gathered for subsequent analyses.

\section{RESULTS AND DISCUSSION}

We demonstrate the potential usefulness of the Monte Carlo method by varying the junction height $h_{\text {junct }}$ to examine the effect it has on light-emission characteristics. We also consider the effect of varying the value of epoxy index of refraction. We assume that the absorption coefficient for the semiconductor chip is $\alpha=10 \mathrm{~cm}^{-1}$. This corresponds to a weakly absorbing medium, with a characteristic absorption length $1 / \alpha$ of $1000 \mu \mathrm{m}$, or 3.75 times the width of the cubic chip. Note that simulation of a realistic device would require that we use experimentally measured absorption coefficient spectrum $\alpha(E)$ for the LED material. The index of refraction for the semiconductor chip is taken to be 3.1, and for the epoxy, both 1.5 and 1.7 are considered. Figure 2(a) shows the light-extraction efficiency for the LED as functions of the light-emitting junction height, which ranges from 0 to $265 \mu \mathrm{m}$ (the height of the chip is $266.7 \mu \mathrm{m}$, or $1.05 \mathrm{mil}$.), for 
two different values of epoxy index of refraction. Note that the efficiency for the $n=1.5$ case is consistently lower than the $n=1.7$ case by a significant amount. This is because the critical angle at the chipepoxy interface is $29^{\circ}$ for $n=1.5$, and $33^{\circ}$ for $n=1.7$. (In practice, epoxy indices do not exceed 1.51.) In both cases, efficiency is low when the junction is near the bottom of the chip where most of the downward pointing photons generated at the junction are absorbed by the bottom contact. As the junction is raised, the efficiency increases until reaching a maximum at around $h_{\text {junct }}=190 \mu \mathrm{m}$, and then decreases by a small amount. At its maximum, the efficiency is nearly twice the $h_{\text {junct }}=0$ value. Figure 2 (b) shows the emission patterns as functions of azimuthal angle $\theta$ for a particular LED structure after each of the three simulation stages (chip, dish, and lens). The chip emission tends to be broadly distributed, the reflector dish brings all emission to $\theta<90^{\circ}$, and the lens tightly focuses a portion of the emission into $\theta<20^{\circ}$, while scattering the remainder to a much broader distribution.

In summary, we employ a Monte Carlo ray tracing technique to model light-extraction characteristics of light-emitting diodes. By effectively utilizing readily available computational resources and relaxing restrictive assumptions on photon traversal history, our method improves upon available analytical models for estimating light-extraction efficiencies from bare LED chips, and enhances modeling capabilities by realistically treating the various processes which photons can encounter in a packaged LED. Our method is not only capable of calculating extraction efficiencies, but can also provide extensive statistical information on photon extraction processes, and predict LED spatial emission characteristics. Simulations using our method can be performed very rapidly on modern workstations, making it a good candidate for an effective design tool. Several areas of our model could be improved to make it more realistic and versatile; a more detailed account of our model is given elsewhere[5]. We believe that, with some refinement, our method could become a valuable LED design tool.
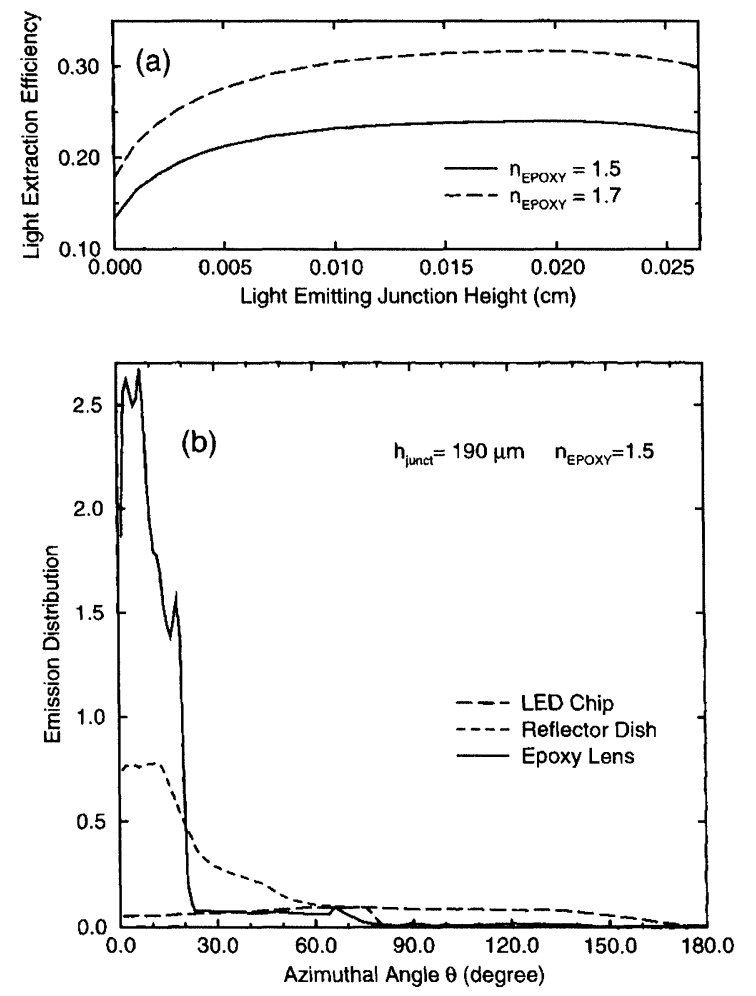

FIGURE 2 (a) Computed photon extraction efficiencies as functions of light-emitting junction height in LED chip for two different epoxy indices of refraction. (b) Azimuthal distributions of photons emitted from a LED structure with $h_{\text {junct }}=190 \mu \mathrm{m}$, and epoxy index of $n=1.5$. Emission patterns after each of the three simulation stages (chip, dish, and lens) are shown

\section{Acknowledgements}

The authors would like to thank J. F. Swenberg, M. W. Wang, F. M. Steranka, M. G. Craford, and P. Mahowald for helpful discussions. This work was supported by the Office of Naval Research (ONR) under Grant No. N00014-92-J-1845. DZT acknowledges partial support from Department of Physics, National Tsing Hua University (Hsinchu).

\section{References}

[1] W. N. Carr, "Photometric figures of merit for semiconductor luminescent sources operating in spontaneous decay mode," Infrared Physics 6, 1-19 (1966).

[2] W. B. Joyce, R. Z. Bachrach, R. W. Dixon, and D. A. Sealer, "Geometrical properties of random particles and the extraction of photons from electroluminescent diodes," J. App. Phys. 45(5), 2229-2253 (1974). 
[3] D. Badouel, "An efficient ray-polygon intersection," in Graphics Gems, A. S. Glassner, Ed., pp.390-399, Academic Press, Boston, MA (1990).

[4] See, for example, E. Hecht, Optics, 2nd Ed., Addison-Wesley, Reading, MA (1987).

[5] D. Z.-Y. Ting and T. C. McGill, "Monte Carlo simulation of LED light-extraction characteristics," to appear in Optical Engineering 34(12) (1995).

\section{Biographies}

David Z.-Y. Ting is an Associate Professor of Physics at the the National Tsing Hua University in Hsinchu, Taiwan, ROC. His research activities include the theoretical studies of electronic and optical properties of semiconductor alloys, quantum wells, superlattices, and heterostructure tunnel devices, quantum transport in nanostructure, and optical simulations. (E-mail:dzt@phys.nthu.edu.tw)

Thomas C. McGill is the Fletcher Jones Professor of Applied Physics at the California Institute of Technology. His research interests are in the broad area of microstructures for modern electronics, semiconductor physics and devices. 

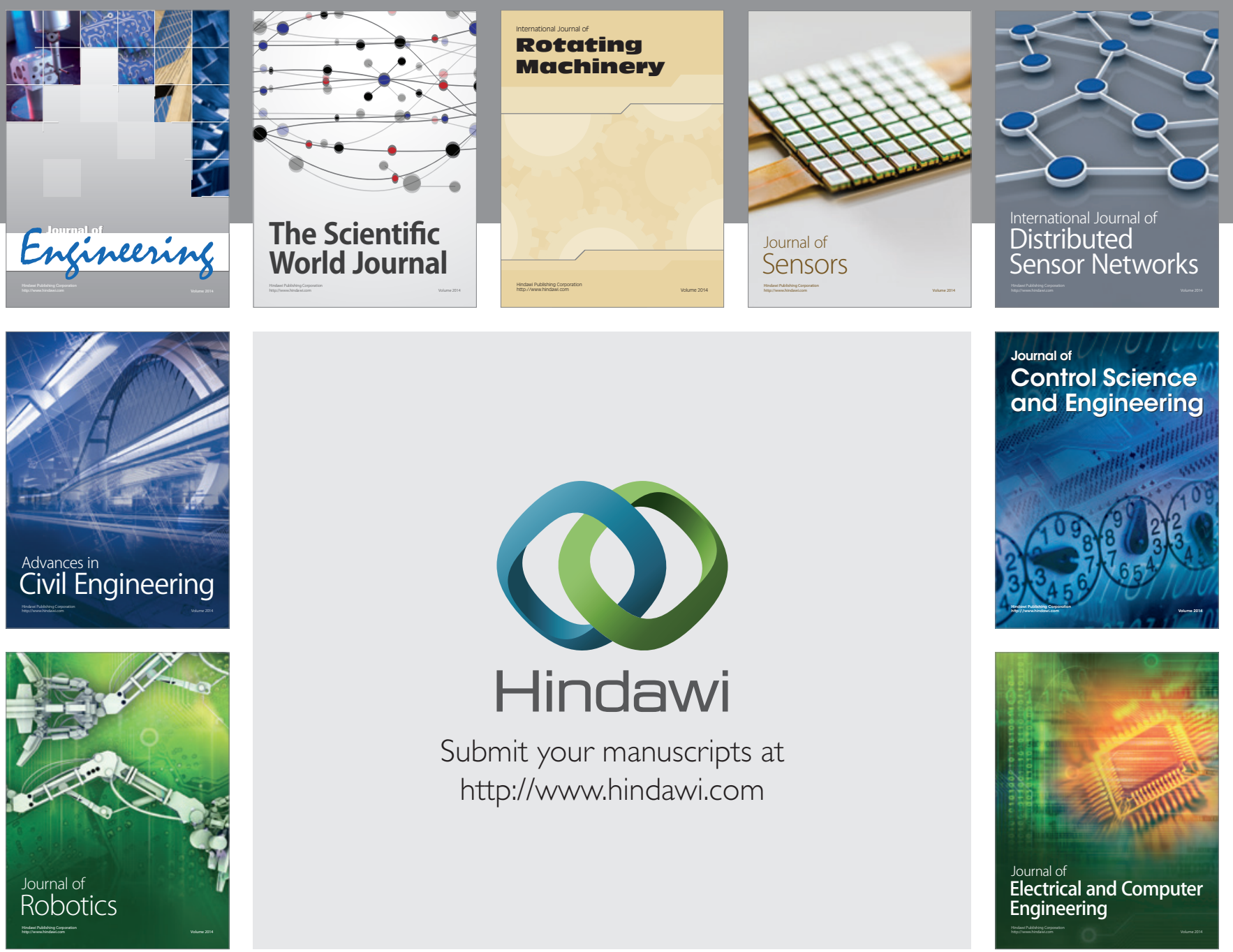

Submit your manuscripts at

http://www.hindawi.com
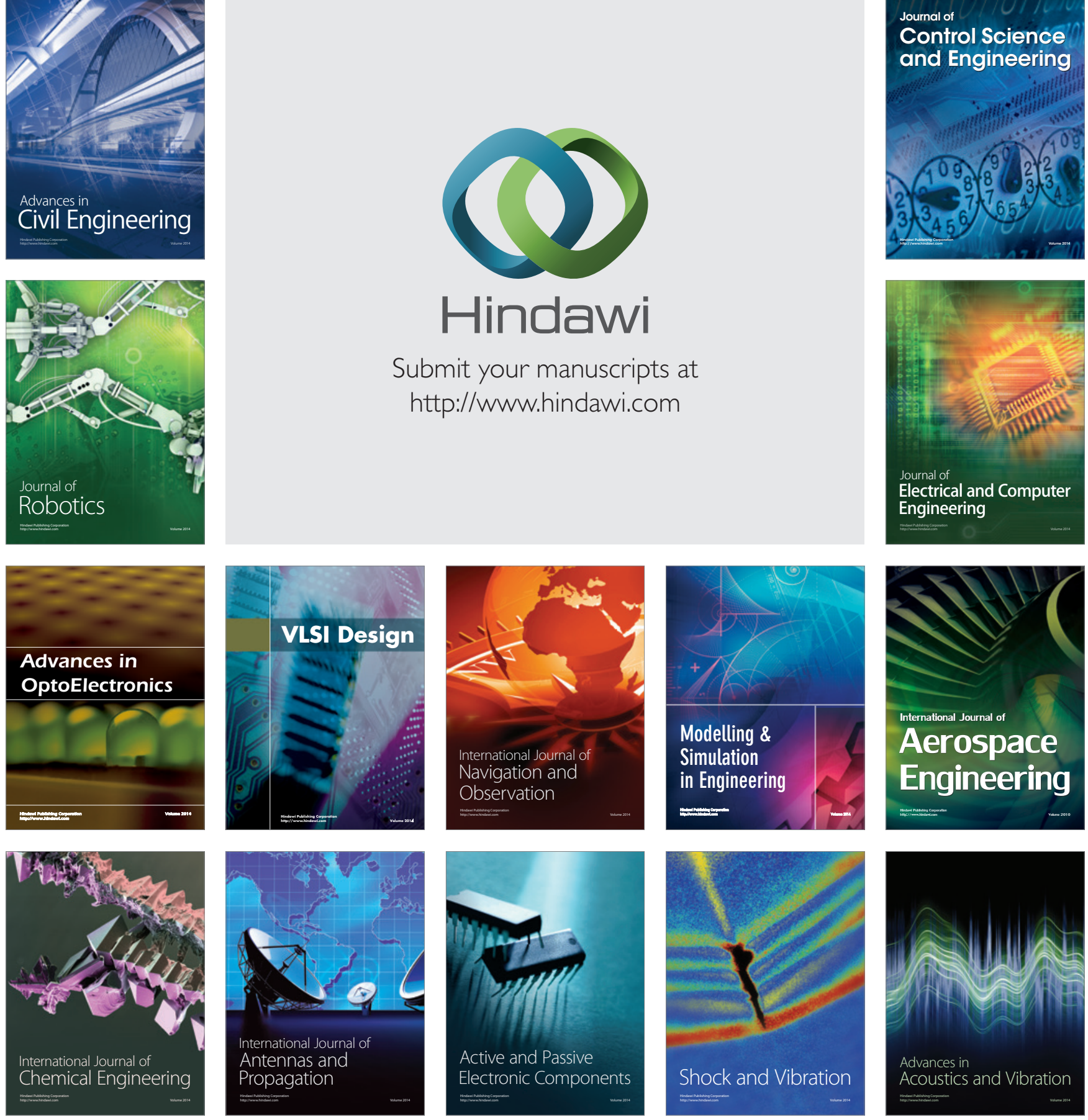